\title{
Spatio-temporal structures in sheared polymer systems
}

\author{
Akira Furukawa and Akira Onuki \\ Department of Physics, Kyoto University, Kyoto 606-8502, Japan
}

\begin{abstract}
We investigate spatio-temporal structures in sheared polymer systems by solving a time-dependent Ginzburg-Landau model in two dimensions. (i) In polymer solutions above the coexistence curve, crossover from linear to nonlinear regimes occurs with increasing the shear rate. In the nonlinear regime the solution behaves chaotically with large-amplitude composition fluctuations. A characteristic heterogeneity length is calculated in the nonlinear regime. (ii) We also study dynamics of shear-band structures in wormlike micellar solutions under the condition of fixed stress. The average shear rate exhibits large temporal fluctuations with occurrence of large disturbances in the spatial structures.
\end{abstract}

Key words: Two-fluid model, Shear-induced concentration fluctuations, Stress-diffusion coupling, Shear-banding, Rheological chaos.

\section{Introduction}

In complex fluids such as polymer solutions, liquid crystals, or surfactant systems, the rheological properties can be very singular and complex. Application of flow field to complex fluids often gives rise to dramatic structural changes, sometimes coupled to phase separation [1,2]. Even chaotic spatio-temporal structures have been observed far away from equilibrium.

In this paper we will present numerical results on two sheared polymer systems: (i) In polymer solutions above the coexistence curve, application of flow has been observed to induce strong composition heterogeneities $[3,4,5,6,7,8,9,10]$. The origin is now ascribed to a dynamical coupling between stress and diffusion in asymmetric viscoelastic mixtures [11,12,13,14]. This is in marked contrast to shear-induced homogenization or mixing observed in near-critical fluids $[15,16]$ and in polymer mixtures with small entanglement effects $[17,18]$. Because some numerical results have already been presented $[19,20,21,22]$, we

Preprint submitted to Elsevier Science 28 October 2018 
will here focus our attention on crossover from linear to nonlinear regimes with increasing the shear rate and chaotic behavior in the nonlinear regime. Furthermore, viscoelastic phase separation below the coexistence curve in shear flow has recently been studied, where polymer-rich domains are fragmented like broken gels [20]. (ii) Next we will briefly examine shear effects in wormlike micellar solutions where the stress-strain relation is nonmonotonic. Wormlike micells have significant chain flexibility and can break and combine [23]. In such systems shear bands composed of low-shear regions and high-shear regions parallel to flow have been observed [24,25,26,27] and rheological chaos, which occurs at low Reynolds numbers, has been discussed experimentally $[28,29,30,31,32]$ and theoretically $[33,34,35,36]$. In the presence of shear bands macroscopic observables, such as the shear stress at fixed shear rate or the shear rate at fixed shear stress, exhibit irregular nonstationary chaotic behavior, which arises from large-scale disturbances of the shear bands. However, not enough understanding has yet been attained on such nonstationary behavior of the shear bands. In this paper we use the word chaotic loosely without rigorous characterization.

Our numerical simulations will be performed in a Ginzburg-Landau scheme, where a conformation tensor representing the chain deformations is a dynamic variable. In previous papers numerical simulations (still in two dimensions) in this line have provided useful insights into the complex nonlinear shear effects $[19,20,21,22]$. The organization of this paper is as follows. We will explain our theoretical scheme in Section 2. We will then present numerical results for polymer solutions in Section 3 and for wormlike micellar solutions in Section 4.

\section{Model equations in dimensionless forms}

Phase behavior of polymer solutions is usually described in terms of the polymer volume fraction $\phi$ only [37]. However, to describe the viscoelastic effects, it is convenient to introduce a symmetric tensor $\stackrel{\leftrightarrow}{W}=\left\{W_{i j}\right\}$ which represents chain conformations undergoing deformations. The free energy density $f$ then depends on $\phi$ and $\stackrel{\leftrightarrow}{W}$. For simplicity, we will measure the free energy density and the stress in units of $\sigma_{0}=k_{\mathrm{B}} T / v_{0} N^{3 / 2}$ where $v_{0}$ is the monomer volume and $N$ is the monomer number on a chain. In terms of the scaled polymer density $\Phi=N^{1 / 2} \phi, f$ reads

$$
f=\Phi \ln \Phi-\frac{u}{2} \Phi^{2}+\frac{1}{6} \Phi^{3}+\frac{2}{\Phi}|\nabla \Phi|^{2}+\frac{g}{4} \Phi^{3} Q(\stackrel{\leftrightarrow}{W})
$$

in the semidilute region $\phi \ll 1$ close to the coexistence curve (in theta solvent). The space integral $F=\int d \boldsymbol{r} f$ gives the free energy. The first three terms 
constitute the Flory-Huggins free energy and the fourth term represents the gradient free energy, where $u$ is related to the interaction parameter $\chi$ by $u=N^{1 / 2}(2 \chi-1)$ [37]. We have $u=2$ and $\Phi=1$ at the critical point and $u=$ $\Phi+\Phi^{-1}$ on the spinodal curve. We measure space in units of $\ell=b N^{1 / 2} / 2 \sqrt{18}$ ( $\sim$ gyration radius), where $b=v_{0}^{1 / d}$ is the monomer length with $d$ being the space dimensionality. The equilibrium average of $W_{i j}$ is the unit tensor $\delta_{i j}$ and the deviation $\delta W_{i j}=W_{i j}-\delta_{i j}$ gives rises to an increase in the free energy. We adopt the Gaussian form $Q(\stackrel{\leftrightarrow}{W})=\sum_{i j}\left(\delta W_{i j}\right)^{2}$. The coefficient $g$ is of order 1 in theta solvent and will be set equal to 1.

Next we derive the dynamic equations using a two-fluid model. We assume that the polymer and the solvent have different velocities, $\mathbf{v}_{p}$ and $\mathbf{v}_{s}$, respectively. The continuity equation is written as $\partial \phi / \partial t=-\nabla \cdot\left(\phi \boldsymbol{v}_{\mathrm{p}}\right)$. In the semidilute region it is rewritten as

$$
\frac{\partial}{\partial t} \Phi+\nabla \cdot(\Phi \boldsymbol{v})=-\nabla \cdot(\Phi \boldsymbol{w})
$$

in terms of the volume-averaged velocity $\mathbf{v}=\phi \mathbf{v}_{p}+(1-\phi) \mathbf{v}_{s}$ and the relative velocity $\boldsymbol{w}=\mathbf{v}_{p}-\mathbf{v}_{s}$. For $\phi \ll 1$ we have $\mathbf{v}_{s} \cong \mathbf{v}$ and $\mathbf{v}_{p} \cong \mathbf{v}+\boldsymbol{w}$. The right hand side of Eq.(2) represents the diffusion. We assume that the solution is incompressible or $\nabla \cdot \boldsymbol{v}=0$. The motion of the conformation tensor $\overleftrightarrow{W}$ is driven by the polymer velocity $\boldsymbol{v}_{p}$ as $[1,22,38,39,40,41]$

$$
\begin{aligned}
& \left(\frac{\partial}{\partial t}+\mathbf{v}_{p} \cdot \nabla\right) W_{i j}-\sum_{k}\left[a\left(S_{i k} \delta W_{k j}+\delta W_{i k} S_{k j}\right)+\left(\delta W_{i k} \Omega_{k j}-\Omega_{i k} \delta W_{k j}\right)\right] \\
& =\left(\nabla_{j} v_{\mathrm{p} i}+\nabla_{i} v_{\mathrm{p} j}\right)-\left(1 / \tau-\mathcal{D} \nabla^{2}\right) \delta W_{i j}
\end{aligned}
$$

where $\nabla_{j}=\partial / \partial x_{j}, S_{i j}=\left(\nabla_{i} v_{\mathrm{p} j}+\nabla_{j} v_{\mathrm{p} i}\right) / 2, \Omega_{i j}=\left(\nabla_{i} v_{\mathrm{p} j}-\nabla_{j} v_{\mathrm{p} i}\right) / 2$, and $\tau$ is the very long rheological time. The left hand side is the frame-invariant time derivative of the tensor $\delta W_{i j}$ and $|a| \leqq 1$ will be assumed in Section 4 . The $\mathcal{D}$ is a kinetic coefficient originally introduced to explain shear banding in the Johnson-Segalman model $[39,40]$, but is not indispensable in the presence of the gradient term in the free energy Eq.(1) for the composition. In our previous papers we have assumed $a=1$ and $\mathcal{D}=0$ for polymer solutions. However, we will assume $a=0.4$ and $\mathcal{D}=1$ for wormlike micellar solutions. The network (viscoelastic) stress due to $\delta W_{i j}$ consistent with Eq.(3) is given by $[2]$

$$
\sigma_{\mathrm{p} i j}=G \sum_{k} \delta W_{i k}\left(\delta_{k j}+a \delta W_{k j}\right)+\frac{1}{4} G Q \delta_{i j}
$$


where $G=g \Phi^{3}$ has the meaning of the shear modulus (in units of $\sigma_{0}$ ) for small rapid deformations. The linear viscosity is given by $\eta=\eta_{0}+G \tau$ in homogeneous states under weak steady shear, which is taken to be much larger than the solvent viscosity $\eta_{0}$. Note that if $G \gg 1$, large viscoelastic effects are produced even for small $\delta W_{i j}$. The eigenvalues of $\delta W_{i j}$ remain small (at most of order 0.1) in our simulations [20], justifying the Gaussian form of $Q$. Physically, this means that the chains are weakly stretched in shear flow. In our case we simply have $\sigma_{\mathrm{p} i j} \cong G \delta W_{i j}$ from Eq.(4).

Hereafter time will be measured in units of $\tau_{0}=\eta_{0} / 4 \sigma_{0}\left(\sim 6 \pi \eta_{0} \ell^{3} / k_{\mathrm{B}} T\right)$. The velocities will be measured in units of $\ell / \tau_{0}=4 \sigma_{0} \ell / \eta_{0}$. If we neglect the inertial effects (accelerations) in the two-fluid model, the velocities $\boldsymbol{w}$ and $\boldsymbol{v}$ read [20]

$$
\boldsymbol{w}=-\nabla \frac{\delta F}{\delta \Phi}+\frac{1}{\Phi} \nabla \cdot \stackrel{\leftrightarrow}{\sigma}_{\mathrm{p}}-\frac{3}{4} g Q \Phi \nabla \Phi, \quad \boldsymbol{v}=\langle\boldsymbol{v}\rangle-\frac{1}{4 \nabla^{2}}[\Phi \boldsymbol{w}]_{\perp}
$$

in the semidilute region. The $\langle\boldsymbol{v}\rangle$ is the mean shear flow, $[\cdots]_{\perp}$ denotes taking the transverse part (whose Fourier component is perpendicular to the wave vector) and the inverse operation $1 / \nabla^{2}$ may be expressed in terms of the Oseen tensor in the limit of large system size [2]. The stress force $\nabla \cdot \overleftrightarrow{\sigma}_{\mathrm{p}}$ in the diffusion flux $\Phi \boldsymbol{w}$ gives rise to the so-called stress-diffusion coupling $[2,14]$. This means that diffusion is induced by stress imbalance $\nabla \cdot \overleftrightarrow{\sigma}_{\mathrm{p}} \neq \mathbf{0}$, which leads to various viscoelastic effects such as nonexponential decay in dynamic light scattering or shear-induced phase separation.

We now have a closed set of dynamic equations for $\Phi$ and $\stackrel{\leftrightarrow}{W}$. In particular, use of these equations yields the time derivative of the free energy $F$,

$$
\frac{d F}{d t}=\int d \boldsymbol{r}\left[\frac{\delta F}{\delta \Phi} \frac{\partial \Phi}{\partial t}+\frac{G}{2} \sum_{i j} \delta W_{i j} \frac{\partial W_{i j}}{\partial t}\right]=\int d \boldsymbol{r}\left[-\Phi^{2} \boldsymbol{v}_{\mathrm{p}} \cdot \boldsymbol{w}-\frac{G}{2 \tau} Q\right]
$$

If $\langle\boldsymbol{v}\rangle=\mathbf{0}$, we have $d F / d t \leqq 0$. The steady condition $d F / d t=0$ is realized in equilibrium where $\delta F / \delta \Phi=$ const. and $\delta W_{i j}=0$. This is a self-consistent condition of dynamic equations which have stable equilibrium solutions [2]. Under this condition we may add the Langevin thermal noise terms in the dynamic equations Eqs.(2) and (3), although they have been omitted for simplicity.

Assuming that the flow direction is along the $x$ axis and the velocity-gradient direction is along the $y$ axis, we give the expressions for the shear stress $\sigma_{x y}$ and the normal stress difference $N_{1}=\sigma_{x x}-\sigma_{y y}$ in units of $\sigma_{0}$ [20],

$$
\begin{aligned}
\sigma_{x y} & =\sigma_{\mathrm{p} x y}-4 \Phi^{-1} \nabla_{x} \Phi \nabla_{y} \Phi+4\left(\nabla_{x} v_{y}+\nabla_{y} v_{x}\right) \\
N_{1} & =N_{\mathrm{p} 1}-4 \Phi^{-1}\left(\nabla_{x} \Phi \nabla_{x} \Phi-\nabla_{y} \Phi \nabla_{y} \Phi\right)+8\left(\nabla_{x} v_{x}-\nabla_{y} v_{y}\right)
\end{aligned}
$$


with $\boldsymbol{v}$ in units of $\ell / \tau_{0}$. The first terms are the network stress components, the second ones arises from the gradient free energy (yielding the surface tension contributions in two-phase states), and the third ones are the viscous contributions. In homogeneous states with weak shear $\dot{\gamma}=\left\langle\nabla_{y} v_{x}\right\rangle$ we find $\sigma_{x y}=\left(g \Phi^{3} \tau+4\right) \dot{\gamma}$ with $\tau$ in units of $\tau_{0}$, so $\eta / \eta_{0}=1+g \Phi^{3} \tau / 4 \gg 1$. For our inhomogeneous flows below, we also have $\left\langle\sigma_{i j}\right\rangle \cong\left\langle\sigma_{\mathrm{p} i j}\right\rangle$ and $\left\langle N_{1}\right\rangle \cong\left\langle N_{\mathrm{p} 1}\right\rangle$.

\section{Nonlinear structures in semidilute polymer solutions}

\subsection{Numerical method for sheared fluids}

For polymer solutions we set $a=1, \mathcal{D}=0$, and $\tau=\tau_{0}\left(0.2+\Phi^{4}\right) /(1+Q)$, where $Q$ in the denominator gives rise to shear-thinning for $\dot{\gamma} \tau \gtrsim 1$ even in homogeneous states $[2,20]$. For weak chain-stretching in the semidilute region, we have $\tau / \tau_{0} \cong \Phi^{4} \gg 1$. Here we explain our numerical method. We integrate the dynamic equations in two dimensions on a $256 \times 256$ lattice. Our numerical scheme is to use the deformed coordinates,

$$
x^{\prime}=x-\gamma(t) y, \quad y^{\prime}=y,
$$

under the periodic boundary condition $f\left(x^{\prime}, y^{\prime}+L\right)=f\left(x^{\prime}+L, y^{\prime}\right)=f\left(x^{\prime}, y^{\prime}\right)$ for any quantity $f\left(x^{\prime}, y^{\prime}\right)$ in the deformed-space representation [42]. Then we can use the FFT method in the deformed space to calculate $\boldsymbol{v}$ from Eq.(5). Here we take $\gamma(t)=\dot{\gamma} t$ at constant $\dot{\gamma}$. In Section 4 , however, $\gamma(t)$ will be determined such that the average shear stress $\left\langle\sigma_{x y}\right\rangle$ is held at a given constant. As the initial condition at $t=0$ we assign Gaussian random compositions at each lattice point, with mean value $\langle\Phi\rangle$ and variance 0.1 . For $t>0$ we solve the dynamic equations without the Langevin noise terms.

\subsection{Mechanism of shear-induced fluctuation enhancement}

For very small shear rate $\dot{\gamma} \ll \tau^{-1}$ the linear stability analysis predicts the composition fluctuation enhancement above the coexistence curve owing to the stress-diffusion coupling $[11,12,13,14]$. At very long wavelengths the relaxation rate strongly depends on the direction of the wave vector $\hat{\boldsymbol{k}}=k^{-1} \boldsymbol{k}$ as [2]

$$
\Gamma(\boldsymbol{k})=\frac{D k^{2}}{1+k^{2} \xi_{\mathrm{ve}}^{2}}\left[1-\frac{2 \phi}{K_{\mathrm{os}}} \frac{\partial \eta}{\partial \phi} \dot{\gamma} \hat{k}_{x} \hat{k}_{y}\right]
$$


where $D$ is the mutual diffusion constant, $K_{\text {os }}=\sigma_{0}\left(\Phi-u \Phi^{2}+\Phi^{3}\right)$ is the osmotic modulus, and $\xi_{v e}\left(\sim\left(\eta / \eta_{0}\right)^{1 / 2} b / \phi\right)$ is the viscoelastic length much longer than the correlation length $(\sim b / \phi)$. The reduction of the relaxation rate (growth rate in spinodal decomposition) or the Onsager kinetic coefficient by the factor $1 /\left(1+k^{2} \xi_{\mathrm{ve}}^{2}\right)$ has been detected by scattering experiments without shear [43,44]. Note that $\Gamma(\boldsymbol{k})<0$ in the direction of $\hat{k}_{x}=\hat{k}_{y}$ for $K<\dot{\gamma} \phi \partial \eta / \phi$. In the semidilute region $\partial \ln \eta / \partial \ln \phi \sim 6$ experimentally ( 7 in our simulation since $\eta \cong G \tau)$. It then follows the condition of strong fluctuation enhancement,

$$
\dot{\gamma}>K_{\mathrm{os}} / 6 \eta \sim 0.1 \tau^{-1}
$$

in fair agreement with a scattering experiment [6]. Under Eq.(10) the linear theory cannot be used and we encounter a nonlinear regime. The orign of the fluctuation enhancement is as follows: when the viscoelastic properties strongly depend on $\phi$, appearance of inhomogeneities can lower the network free energy ( $\propto g$ in Eq.(1)) and the stress (at fixed shear rate). By the same reason, when long and short polymers pass through a capillary tube, the longer ones migrate toward the tube center [14].

\subsection{Shear-induced fluctuations above the coexistence curve}

Figure 1 shows time evolution of the variance $\sqrt{\left\langle(\delta \Phi)^{2}\right\rangle(t)}$, the shear stress $\left\langle\sigma_{x y}\right\rangle(t)$, and the normal stress difference $\left\langle N_{1}\right\rangle(t)$ after application of shear at $t=0$ for $\dot{\gamma}=0.05 \cong 0.8 / \tau$. The averages are taken over all the lattice points. Here the system is above the coexistence curve with $u=2$ and $\langle\Phi\rangle=2$. Though there is no thermal noise in the dynamic equations, the variance saturates at about 0.6 in the late stage. Figure 2 suggests that the system is in a state of deterministic chaos, undergoing partial or incomplete phase separation. Both $\left\langle\sigma_{x y}\right\rangle(t)$ and $\left\langle N_{1}\right\rangle(t)$ exhibit a pronounced peak at the onset of fluctuation enhancement at $t \sim 25$ and noisy behavior follows in the subsequent dynamical steady state. The noise of these quantities arises from breakup of the polymer-rich domains supporting the stress. The effective viscosity $\eta_{\text {eff }}=\left\langle\sigma_{x y}\right\rangle / \dot{\gamma}$ in the steady state is about 8 times larger than the solvent viscosity $\eta_{0}$. It would be $28 \eta_{0}$ for the homogeneous case, so marked shear thinning is realized. For comparison the shear stress (upper broken line) and the normal stress difference (lower broken line) are calculated for the homogeneous case.

Figure 2 displays the normalized composition $\Phi(\boldsymbol{r}, t)$ at $t=45$ (left) and $t=300$ (right) in the run which produced Fig.1. In the top figures we cannot see well-defined interfaces, but the composition changes over a mesoscopic, characteristic length $R_{\mathrm{h}}$ in the steady state. We numerically find that the average gradient term $\left\langle 2|\nabla \Phi|^{2} / \Phi\right\rangle$ and the average elastic energy $\left\langle g \Phi^{3} Q / 4\right\rangle$ in 


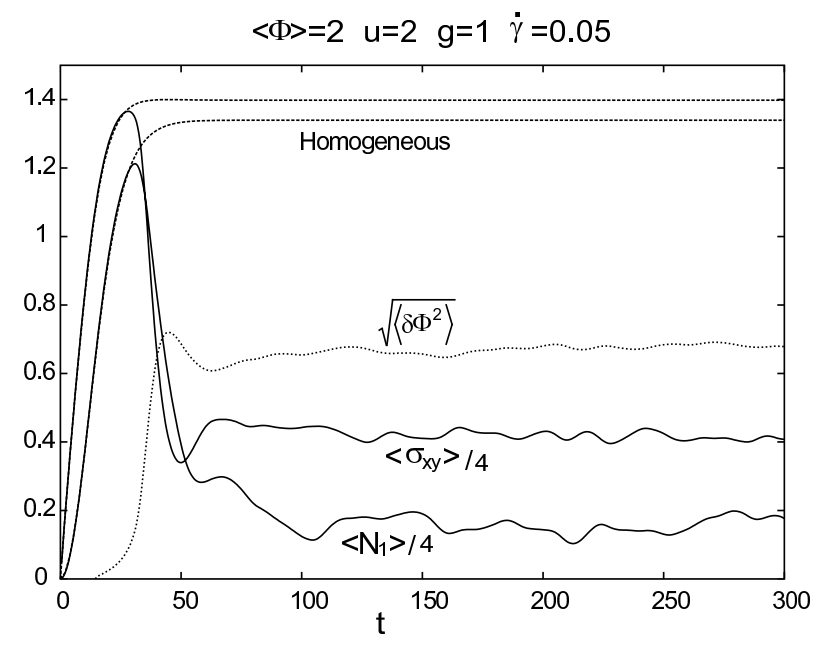

Fig. 1. Time evolution of $\sqrt{\left\langle\delta \Phi^{2}\right\rangle}$ (dotted line), $\left\langle\sigma_{x y}\right\rangle$, and $\left\langle N_{1}\right\rangle$ (solid lines) after application of shear. At $t=0$, the system is above the coexistence curve. The shear-induced fluctuations (shown in Fig.2) give rise to the pronounced stress overshoots. The upper curves are those for the homogeneous case.

the dimensionless free energy density Eq.(1) are of the same order for various $\dot{\gamma}$ and $\langle\Phi\rangle$. We estimate the former as $\langle\Phi\rangle / R_{\mathrm{h}}^{2}$ and the latter as $\left\langle\sigma_{x y}\right\rangle^{2} /\langle\Phi\rangle^{3}$ to obtain

$$
R_{\mathrm{h}} \sim\langle\Phi\rangle^{2} /\left\langle\sigma_{x y}\right\rangle
$$

or $R_{\mathrm{h}} \sim b G / \phi\left\langle\sigma_{x y}\right\rangle$ in the original units. The behavior of $\left\langle\sigma_{x y}\right\rangle$ as a function of $\dot{\gamma}$ can be known from Fig.5. On the other hand, to the stress, the nework contributions are mostly much larger than the gradent contributions in Eqs.(7)

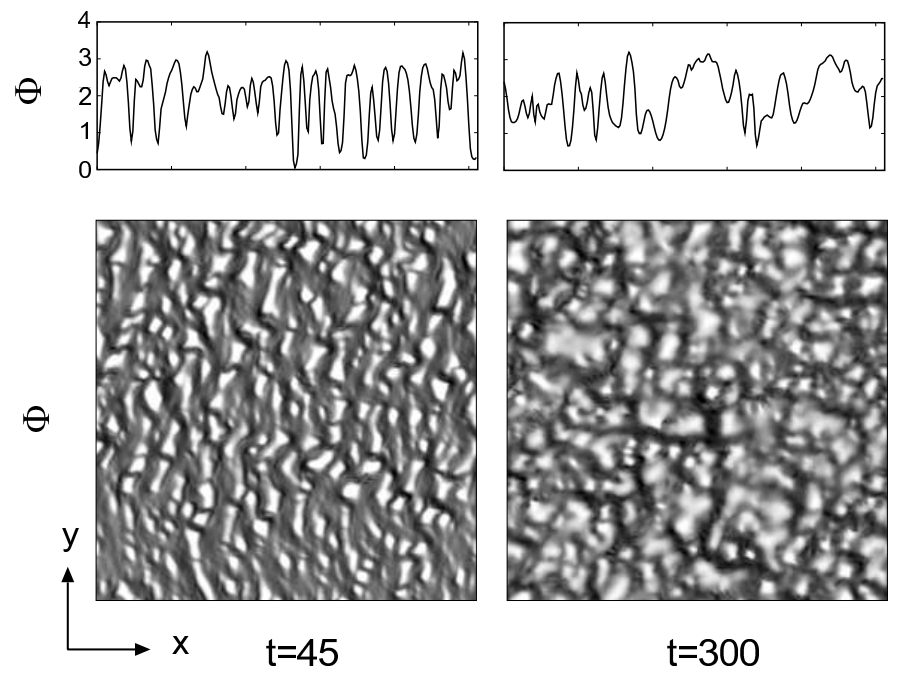

Fig. 2. $\Phi(x, y, t)$ at $t=45$ (left) and $t=300$ (right) above the coexistence curve. The top figures are the profiles along the $x$ axis at $y=128$. The parameters are the same as those in Fig.1. For $t>100$ a dynamical steady state is realized. 
and (8).

The length $R_{\mathrm{h}}$ should be measurable by scattering. In Fig.3 the time-averaged structure factor $S\left(k_{x}, k_{y}\right)=\left\langle\left|\Phi_{\mathbf{k}}\right|^{2}\right\rangle$ in the dynamical steady state is shown. It has sharp peaks in the $k_{x}-k_{y}$ plane close to the $k_{x}$ axis, presumably at $k_{x} \sim \pm 2 \pi / R_{\mathrm{h}}$. This is in agreement with the experimental structure factor for $\dot{\gamma} \tau \sim 1$ in the $k_{x}-k_{y}$ plane [3]. It is worth noting that the light scattering in the $k_{x}-k_{z}$ plane was also intensified along the $k_{x}$-axis for $\dot{\gamma} \tau \sim 1$ [6]. Microscope pictures in the $x-z$ plane of the shear-induced composition fluctuations closely resemble the pattern at $t=300$ in Fig.2 [8]. These suggest stronger correlations perpendicular to the flow direction than along the flow for $\dot{\gamma} \tau \sim 1$. Below the coexistence curve also, the structure factor exhibits similar behavior [20].
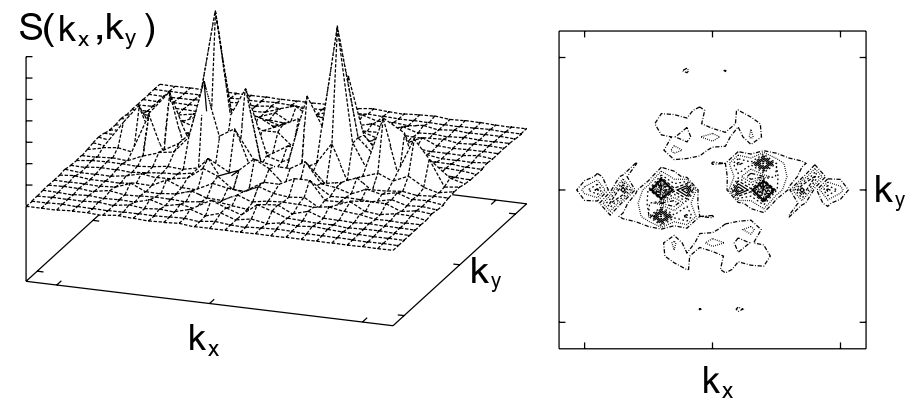

Fig. 3. Bird-view (left) and contour plot (right) of the time-averaged structure factor $S\left(k_{x}, k_{y}\right)=\left\langle\left|\Phi_{\mathbf{k}}\right|^{2}\right\rangle$ in the dynamical steady state in Fig.2.

\subsection{Chaotic behavior above the coexistence curve}

As shown in Fig.4, there emerge two dynamic regimes at longer times. When $\dot{\gamma}$ is smaller than a characteristic shear rate $\dot{\gamma}_{\mathrm{nl}}(\cong 0.01$ for $\langle\Phi\rangle=2)$, the fluctuations enhanced in the early stage slowly relax to zero due to the convective deformation by shear in the absence of the thermal noise. In this linear or thermal regime, the steady-state fluctuations can be attained only in the presence of the thermal noise. It should be noted that in the previous theories the fluctuation intensity was calculated in the linear approximation in the presence of the thermal noise. $[11,12,13,14]$. In the nonlinear or chaotic regime $\dot{\gamma}>\dot{\gamma}_{\mathrm{nl}}$, the large-amplitude fluctuations are continuously produced by the nonlinear interactions among them even without the thermal noise. In Fig. 5 we show a discontinuous change in the steady-state variance with varying $\dot{\gamma}$ for three $\langle\Phi\rangle$ (left), which occurs roughly at $\dot{\gamma} \sim 0.2 / \tau$ with $\tau \cong \tau_{0}\left(0.2+\Phi^{4}\right)$. The variance goes to zero in the linear regime in the absence of the thermal noise. Of course, the changeover becomes continuous in the presence of the thermal noise. In Fig.5 we also show the normalized effective viscosity $\eta_{\text {eff }} / \eta_{0}=\left\langle\sigma_{x y}\right\rangle / \eta_{0} \dot{\gamma}$ versus $\dot{\gamma}$ (right), which exhibits Newtonian behavior in the linear regime and strong shear-thinning behavior with $\eta_{\text {eff }} \sim 1 / \dot{\gamma}$ in the nonlinear regime. 


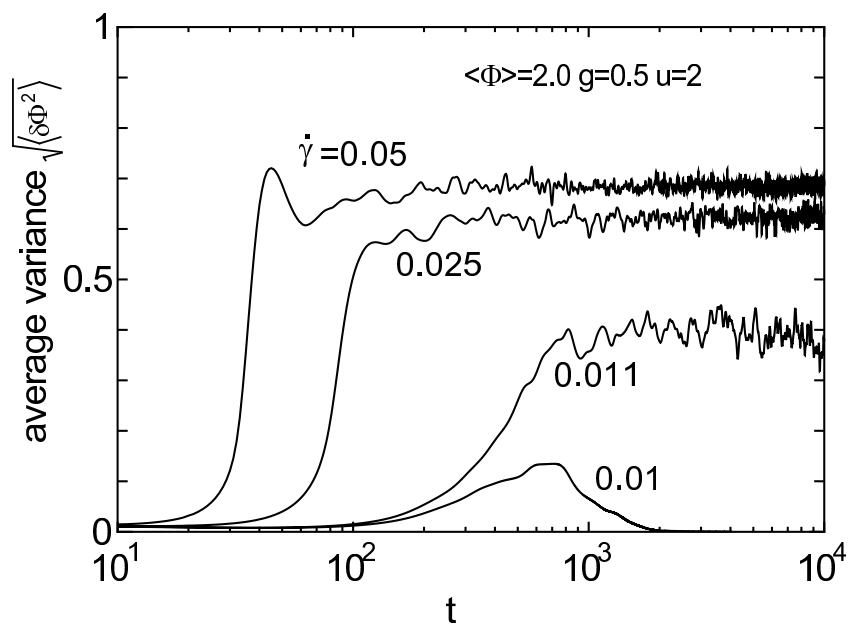

Fig. 4. Time evolution of $\sqrt{\left\langle(\delta \Phi)^{2}\right\rangle}(t)$ for four values of $\dot{\gamma}$ without the Langevin noise. A chaotic steady state is realized for the upper three curves. For the smallest shear $\dot{\gamma}=0.01$ the fluctuations enhanced in the early stage slowly relax to zero.
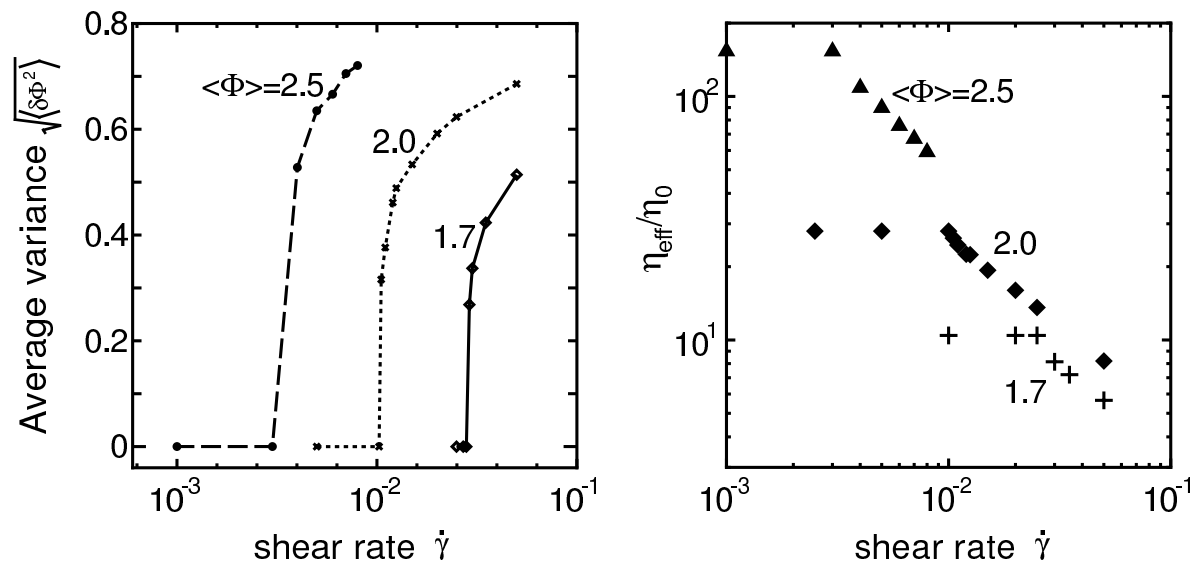

Fig. 5. Steady-state variance $\sqrt{\left\langle(\delta \Phi)^{2}\right\rangle}$ (left) and normalized effective viscosity $\eta_{\text {eff }} / \eta_{0}$ (right) versus $\dot{\gamma}$ for three values of $\langle\Phi\rangle$. Here there is no Langevin noise terms and a sharp discontinuous transition occurs between the linear and nonlinear regimes. Shear-thinning behavior is marked in the nonlinear regime.

We give some remarks. (i) In one-phase states of near-critical fluids without viscoelasticity, the critical fluctuations are always suppressed by shear flow below the equilibrium level and the thermal noise is indispensable to produce the steady-state structure factor $[2,15]$. (ii) Below the coexistence curve, on the contrary, the random noise terms are generally irrelevant in producing large-scale phase-separation patterns and are usually omitted in simulations without and with shear flow [2]. However small the shear rate is, the domains are eventually deformed nonlinearly by applied shear flow as the domains grow. Therefore, the linear shear regime is nonexistent in late-stage phase separation. For Newtonian fluid mixtures with small surface tension, stringlike domain structures can be formed in steady states [18]. (iii)The system considered here has very low Reynolds numbers, so that the fluid inertia plays 
no role in the irregular patterns.

\section{Nonlinear structures in wormlike micellar solutions}

\subsection{Nonmonotonic stress-strain relation}

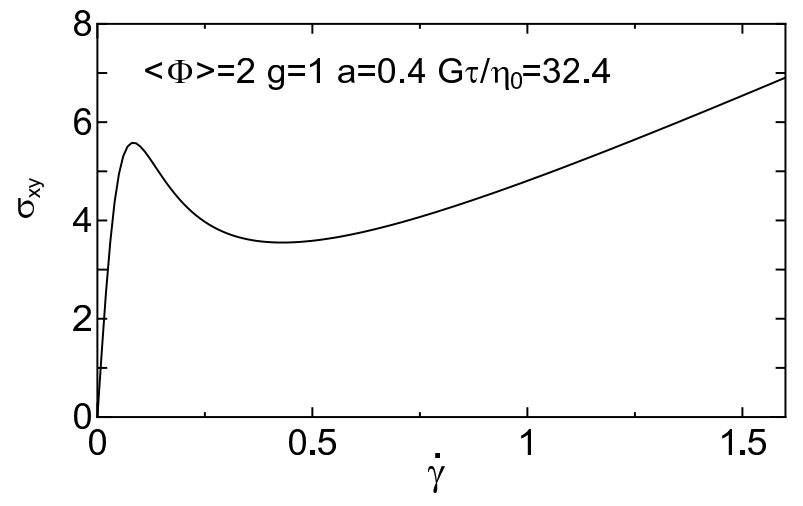

Fig. 6. Stress-strain relation Eq.(12) for wormlike micellar solutions in homogeneous states. The region $\partial \sigma_{x y} / \partial \dot{\gamma}<0$ is unstable and shear bands appear.

We present preliminary results on sheared wormlike micellar solutions where $a=0.4, \mathcal{D}=1$, and $\tau=\tau_{0}\left(0.2+\Phi^{4}\right)$ in Eq.(3). Several authors have used the framework of the two-fluid dynamics including the composition $[22,41]$. For $|a|<1$ it is important that the stress-strain curve is nonmonotonic. That is, for homogeneous shear flow, Eq.(3) is solved to give $\delta W_{x y}=\dot{\gamma} \tau /\left[1+(\dot{\gamma} \tau)^{2}\left(1-a^{2}\right)\right]$ and $\delta W_{x x} /(a+1)=\delta W_{y y} /(a-1)=\dot{\gamma} \tau \delta W_{x y}$. Then Eq.(4) gives

$$
\sigma_{x y}=\eta_{0} \dot{\gamma}+G \dot{\gamma} \tau \frac{\left[1+(\dot{\gamma} \tau)^{2}\left(1+a^{2}\right)\right]}{\left[1+(\dot{\gamma} \tau)^{2}\left(1-a^{2}\right)\right]^{2}}
$$

For $\eta_{0} / G \tau \ll 1$ the curve has a maximum at $\dot{\gamma} \sim 1 / \tau$ and a minimum at $\dot{\gamma} \sim\left(G / \eta_{0} \tau\right)^{1 / 2}$ for $a$ not very close to 1 . Fig. 6 displays the curve for $G \tau / \eta_{0}=$ 32.4. It is known that homogeneous states are unstable for $\partial \sigma_{x y} / \partial \dot{\gamma}<0$. Shear banding instability occurs in the region of $\sigma_{x y}$ where $\dot{\gamma}$ is multi-valued.

\subsection{Numerical method at fixed stress}

For this system there have been experiments both at constant shear rate $[28,29,30,31]$ and at constant shear stress [32]. Since simulations of a model similar to ours have already been performed at constant shear rate for $a=0$ [22], we here show results at constant shear stress. We solve the dynamic 
equations in Section 2 on a $128 \times 128$ lattice using the numerical scheme in Section 3 [42]. We impose the periodic boundary condition in the deformed space, so the boundary effects do not appear in our scheme. We use the Stokes approximation $\nabla \cdot \stackrel{\leftrightarrow}{\sigma}=\mathbf{0}$ to find $\int_{0}^{L} d x \nabla_{y} \sigma_{x y}(\boldsymbol{r}, t)=0$, where $\stackrel{\leftrightarrow}{\sigma}$ is the (total) stress tensor and $L$ is the system length in the $x$ axis. Then the lateral average defined by

$$
\Sigma=\int_{0}^{L} d x \frac{1}{L} \sigma_{x y}(\boldsymbol{r}, t)
$$

becomes independent of $y$. If $\Sigma$ is a constant independent of time, Eq.(7) yields the average shear rate dependent on time in the dimensionless form,

$$
4\langle\dot{\gamma}\rangle(t)=\Sigma-\left\langle\sigma_{\mathrm{p} x y}-4 \Phi^{-1} \nabla_{x} \Phi \nabla_{y} \Phi\right\rangle
$$

where \langle\rangle denotes taking the space average. At each time step we calculate the average strain $\int_{0}^{t} d t^{\prime}\langle\dot{\gamma}\rangle\left(t^{\prime}\right)$ as the shear strain $\gamma(t)$ in Eq.(9).

\subsection{Chaotic behavior with concentration inhomogeneities at fixed stress}

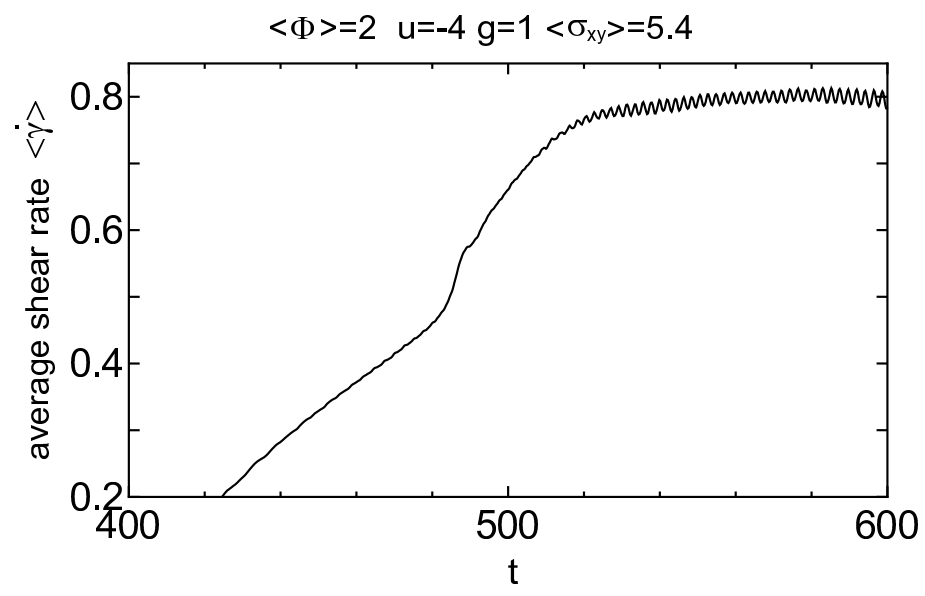

Fig. 7. Time evolution of the average shear rate $\langle\dot{\gamma}\rangle(t)$ for $\left\langle\sigma_{x y}\right\rangle=5.4$ and $\langle\Phi\rangle=2.0$.

Because we set $u=-4$, the system is in a one-phase state before application of shear. Figure 7 presents the time evolution of the average shear rate $\langle\dot{\gamma}\rangle(t)=$ $\left\langle\partial v_{x} / \partial y\right\rangle$ in the initial stage of band formation after application of shear flow at $t=0$. In Fig.8 we show snapshots of $\Phi(\boldsymbol{r}, t)$ and $\dot{\gamma}(\boldsymbol{r}, t)=\nabla_{y} v_{x}$ at various times in this stage. We can see that the homogeneous state becomes unstable at $t \sim 400$ and the shear bands subsequently begin to grow in the time region $t=$ $400 \sim 500$. The pseudo one-dimensional band structures are soon deformed in the flow direction, resulting in the irregular oscillatory behavior of $\langle\dot{\gamma}\rangle(t)$. 

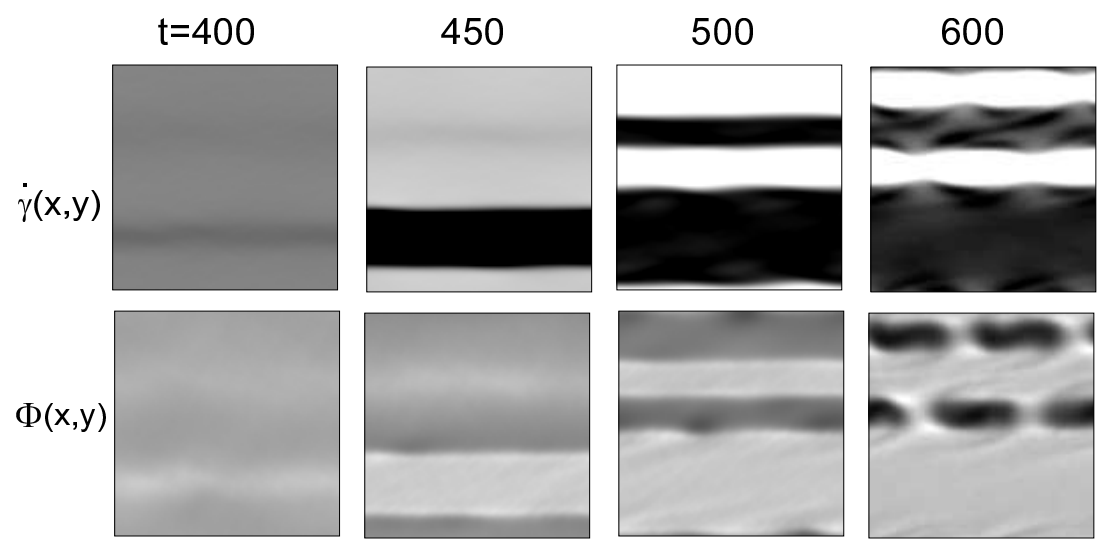

Fig. 8. Snapshots of $\Phi(\mathbf{r}, t)$ and $\dot{\gamma}(\mathbf{r}, t)=\nabla_{y} v_{x}$ at $t=400,450,500,600$. The dark regions take larger values than the bright regions.

We next explain the long-time behavior. Figure 9 shows the chaotic temporal fluctuations of the space average $\langle\dot{\gamma}\rangle(t)=\left\langle\partial v_{x} / \partial y\right\rangle$ for $\left\langle\sigma_{x y}\right\rangle=5.4$ and $\langle\Phi\rangle=$ 2.0. We notice that $\langle\dot{\gamma}\rangle(t)$ frequently exhibits large amplitude fluctuations, which arises from large-scale disturbances of the interfaces between different shear bands. Figure 10 illustrates $\Phi(\boldsymbol{r}, t)$ and $\dot{\gamma}(\boldsymbol{r}, t)=\nabla_{y} v_{x}$ at $t=9000$ (A), $t=14000$ (B), and $t=15250$ (C) in Fig.9. The bird views of (A) and (C) are given also. We find that the system jumps between the two states, (A) and (B), alternatively. In these states the thickness of the low-shear band (white) is considerably different. Furthermore, at $t \sim 15000$ in $(\mathrm{C})$ the interface between two bands becomes globally unstable, resulting in abrupt expansion of the low-shear band. We can see that the velocity and the composition are strongly coupled. Thus these chaotic spatio-temporal behavior arise from the stress-diffusion coupling in our model.

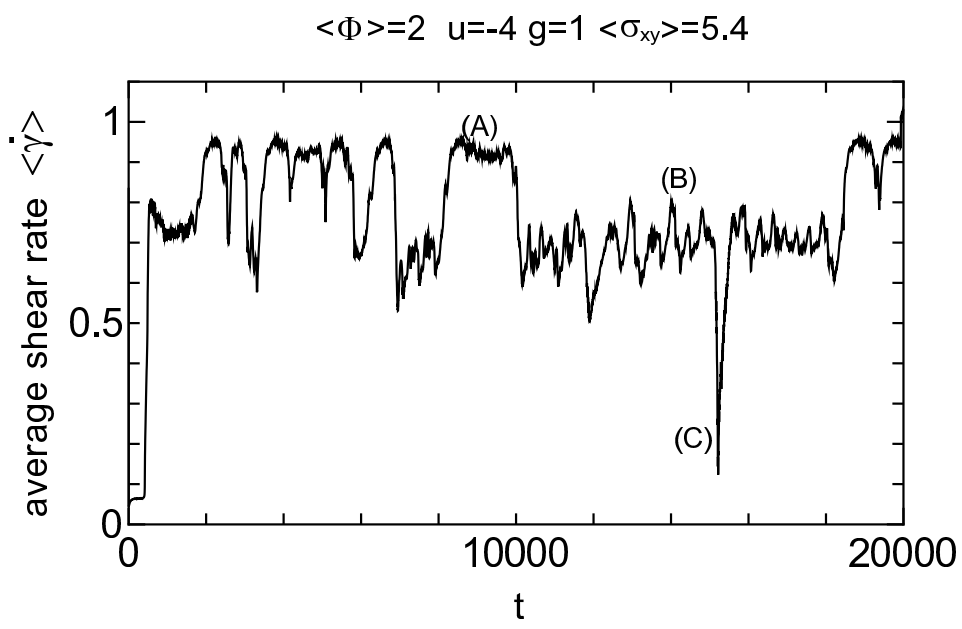

Fig. 9. Time evolution of the average shear rate $\langle\dot{\gamma}\rangle(t)$ for $\left\langle\sigma_{x y}\right\rangle=5.4$ and $\langle\Phi\rangle=2.0$. 


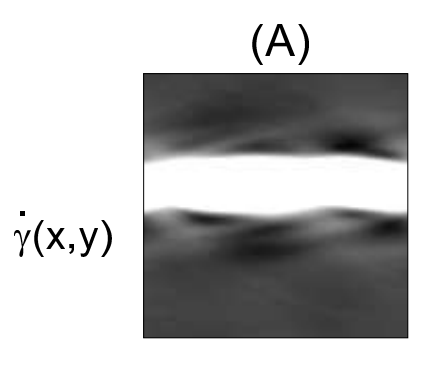

(B)

(C)
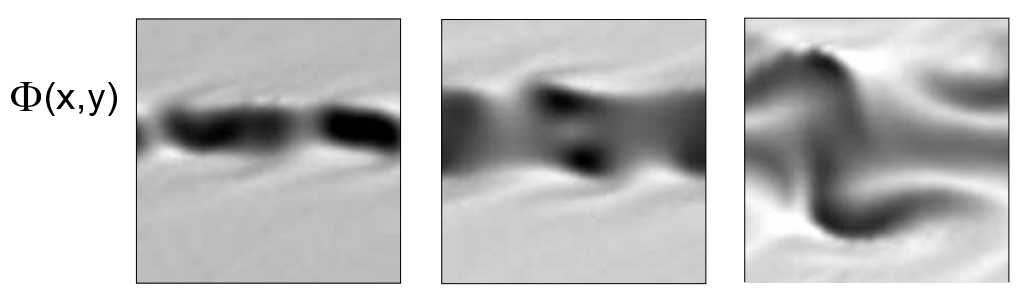

(A)

(C)
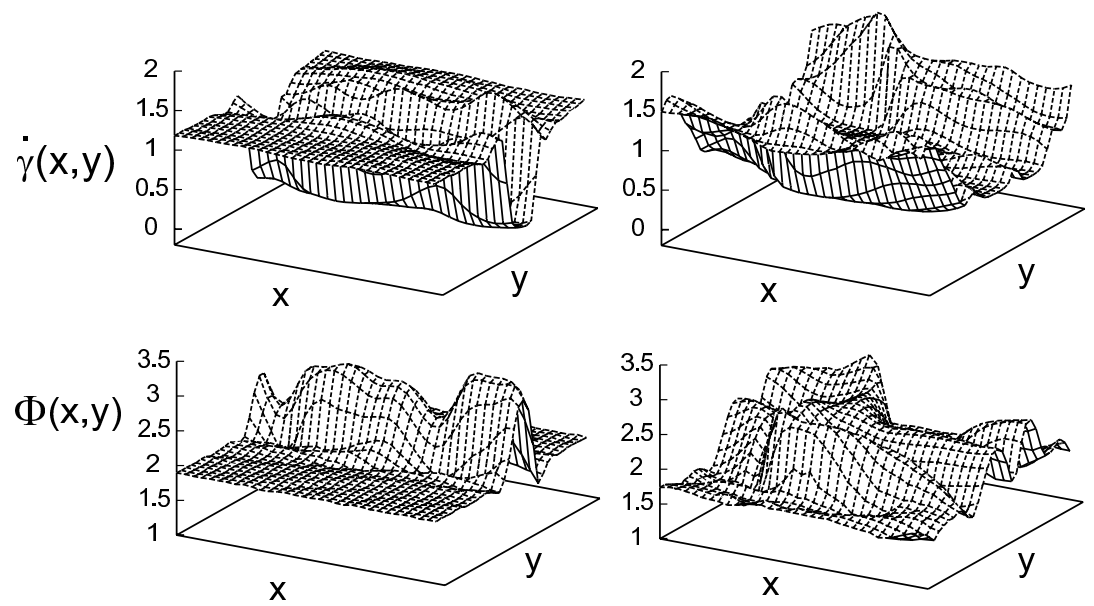

Fig. 10. Snapshots and bird views of $\Phi(\mathbf{r}, t)$ and $\dot{\gamma}(\mathbf{r}, t)=\nabla_{y} v_{x}$ at $t=9000$ (A), $t=14000(\mathrm{~B})$, and $t=15250$ (C) in Fig.9. The dark regions take larger values than the bright regions in the upper snapshots.

\section{Summary}

We have investigated complex spatio-temporal structures in sheared polymer systems by solving a time-dependent Ginzburg-Landau model. Though performed in two dimensions, we have revealed a number of unique features of sheared polymer systems. We summarize our main results.

(i) In polymer solutions above the coexistence curve, Fig.4 shows two dynamic regimes. In the linear regime $\dot{\gamma}<\dot{\gamma}_{\text {nl }}$ the shear-induced fluctuations can be maintained only in the presence of the thermal noise, while in the nonlinear regime $\dot{\gamma}>\dot{\gamma}_{\text {nl }}$ chaotic dynamical steady states are realized due to the nonlinear interactions among the fluctuations. The previous linear calculations $[11,12,13,14]$ are meaningful only in the linear regime, and our results in 
Figs.1-5 are those in the nonlinear regime. The stress overshoot in Fig.1 occurs due to appearance of the shear-induced composition fluctuations in Fig.2. In the dynamical steady states of the nonlinear regime the heterogeneous deviation $\delta \phi$ is even on the order of the average $\langle\phi\rangle$ varying on the scales of the characteristic size $\sim R_{\mathrm{h}}$ as in the top figures of Fig.2. We have related $R_{\mathrm{h}}$ to the average shear stress as in Eq.(12) in the dynamical steady states.

(ii) We have also presented our preliminary results of shear-band structures in wormlike micellar solutions under the condition of fixed stress. As shown in Figs.9 and 10, the average shear rate exhibits large temporal fluctuations. However, we have not yet understood the mechanism why the shear bands exhibit irregular behavior in shear flow.

We will shortly report simulations of wormlike micellar solutions both at fixed shear rate and at fixed shear stress. We are not aware of any previous simulations at fixed shear stress. As other problems not treated here, we mention rheological turbulence at low Reynolds numbers in dilute polymer solutions [45] and defect turbulence in nematic liquid crystalline polymers in shear flow $[2,46,47]$. Both shear banding and tumbling have been observed in suspensions of rods [48]. Liquid crystalline molecules can tumble in shear flow, constituting a coupled oscillator system [49], and loss of their phase coherency leads to proliferation of disclinations. Thus a number of nonequilibrium problems remain largely unexplored in complex fluids, which could also be interesting subjects in nonlinear science and in fluid mechanics.

\section{Acknowledgments}

It is our great pleasure to acknowledge the lasting contribution of Professor Yoshiki Kuramoto in nonlinear science [49]. This work was supported by Grants in Aid for Scientific Research and for the 21st Century COE project (Center for Diversity and Universality in Physics) from the Ministry of Education, Culture, Sports, Science and Technology of Japan.

\section{References}

[1] R.G. Larson, The Structure and Rheology of Complex Fluids (Oxford University Press, 1999); Rheol. Acta. 31, 497 (1992).

[2] A. Onuki, Phase Transition Dynamics (Cambridge University Press, Cambridge, 2002); J. Phys C 9, 6119 (1997).

[3] X.L. Wu, D.J. Pine, and P.K. Dixon, Phys. Rev. Lett. 68, 2408 (1991).

[4] T. Hashimoto and K. Fujioka, J. Phys. Soc. Jpn. 60, 356 (1991); T. Hashimoto and T. Kume, ibid. 61, 1839 (1992). 
[5] A.I. Nakatani, J.F. Douglas, Y.-B. Ban, and C.C. Han, J. Chem. Phys. 100, 3224 (1994).

[6] S. Saito, T. Hashimoto, I. Morfin, P. Linder, and F. Boue, ibid. 35, 445 (2002); M.K. Endoh, S. Saito, and T. Hashimoto, ibid. 35, 7692 (2002).

[7] J. van Egmond, D.E. Werner, and G.G. Fuller, J. Chem. Phys. 96, 7742 (1992).

[8] E. Moses, T. Kume, and T. Hashimoto, Phys. Rev. Lett. 72, 2037 (1994).

[9] I. Morfin, P. Linder, and F. Boue, Macromolecules 32, 7208 (1999).

[10] K. Migler, C. Liu, and D.J. Pine, Macromolecules 29, 1422 (1996).

[11] E. Helfand and G.H. Fredrickson, Phys. Rev. Lett. 62, 2468 (1989).

[12] A. Onuki, Phys. Rev. Lett. 62, 2472 (1989). J. Phys. Soc. Jpn. 59, 3423 (1990).

[13] S.T. Milner, Phys. Rev. E 48, 3674 (1993).

[14] M. Doi and A. Onuki, J. Phys. II 2, 1631 (1992).

[15] A. Onuki and K. Kawasaki, Ann. Phys. (NY) 121, 456 (1979).

[16] D. Beysens and M. Gbadamassi, Phys. Rev. A 22, 2250 (1980); D. Beysens, M. Gbadamassi, and B. Moncef-Bouanz, Phys. Rev. A 28, 2491 (1983).

[17] K. Asakawa and T. Hashimoto, J. Chem. Phys. 105, 5216 (1996); T. Hashimoto, K. Matsuzaka, and K. Fujioka, ibid. 108, 6963 (1998).

[18] T. Hashimoto, K. Matsuzaka, E. Moses, and A. Onuki, Phys. Rev. Lett. 74, 126 (1995).

[19] A. Onuki, R. Yamamoto, and T. Taniguchi, J. Physique II 7, 295 (1997); Progress in Colloid \& Polymer Science 106, 150 (1997).

[20] T. Imaeda, A. Furukawa, and A. Onuki, Phys. Rev. E 70, **(2004).

[21] T. Okuzono, Modern Phys. Lett. 11, 379 (1997).

[22] X. -F. Yuan and L. Jupp, Europhys. Lett. 60, 691 (2002); L. Jupp, T. Kawakatsu, and X.-F. Yuan, J. Chem. Phys. 119, 6361(2003).

[23] M. Cates, Macromolecules 20, 2289 (1987).

[24] J.-F. Berret, D.C. Roux, and G. Porte, J. Phys. II 4, 1261, (1994).

[25] P. Boltenhagen, Y. Hu, E.F. Matthys, and D.J. Pine, Phys. Rev. Lett. 79, 2359 (1997).

[26] P.T. Callaghan, M.E. Cates, C.J. Rofe, and J.B.A.F. Smeulders, J. Phys II 6, 375 (1996).

[27] C. Grand, J. Arrault, and M.E. Cates, J. Phys II 7, 1071 (1997).

[28] F. Pignon, A. Magnin, and j.-M. Piau, J. Rheol. 40, 573, (1996). 
[29] R. Bandyopadhyay, and A.K. Sood, Europhys. Lett. 56, 447 (2001).

[30] W.M. Holmes, M.R. López-González, and P.T. Callaghan, Europhys. Lett. 64, $274(2003)$.

[31] J.-B. Salmon, S. Manneville, and A. Colin, Phys. Rev. E 68, 051504, (2003).

[32] S. Manneville, J.-B. Salmon, and A. Colin, Eur. Phys. J. E, 13, 197, (2004).

[33] M.E. Cates, D.A. Head, and A. Ajdari, Phys. Rev. E 66, 025202, (2002).

[34] G. Picard, A. Ajdari, L. Bocquet, and F. Lequeux, Phys. Rev. E 66, 051501 (2002).

[35] S.M. Fielding and P.D. Olmsted, Phys. Rev. Lett. 92, 084502 (2004).

[36] B. Chakrabarti, M. Das, C. Dasgupta, S. Ramaswamy, and A.K. Sood, Phys. Rev. Lett. 92, 055501 (2004).

[37] P.G. de Gennes, Scaling Concepts in Polymer Physics (Cornell University Press, Ithaca, 1980).

[38] M. Jhonson and D. Segalman, J. Non-Newtonian Fluid Mech., 69, 255, (1977).

[39] X.-F. Yuan, Europhys. Lett., 46, 542 (1999).

[40] C.-Y. David Lu, P.D. Olmsted, and R.C. Ball, Phys. Rev. Lett., 84, 642, (2000).

[41] S.M. Fielding and P.D. Olmsted, Phys. Rev. E, 68, 036313 (2003).

[42] A. Onuki, J. Phys. Soc. Jpn. 66, 1836 (1997).

[43] D. Schwahn, S. Janssen, and T. Springer, J. Chem. Phys. 97, 8775 (1992).

[44] M Takenaka, H. Takeno, H. Hasegawa, S. Saito, T. Hashimoto, and M. Nagao, Phys. Rev. E 65, 021806 (2002).

[45] A. Groisman and V. Steinberg, Nature (London) 405, 53 (2000).

[46] G. Marrucci and F. Greco, in Advances in Chemical Physics, ed. I. Prigogine and S. Rice, (John Wiley \& Sons, 1993), p.331.

[47] R.G. Larson and M. Doi, J.Rheol. 35, 539 (1991).

[48] J.K.G. Dhont, M.P. Lettinga, Z. Dogic, T.A.J. Lenstra, H. Wang, S. Rathgeber, P. Carletto, L. Willner, H. Frielinghaus, and P. Lindner, Faraday Discuss., 123, 157 (2003).

[49] Y. Kuramoto, Chemical Oscillations, Waves, and Turbulence (Springer, New York, 1984). 